\title{
REVIEW ON SAFETY ASSESSMENT OF A COSMETIC PRODUCT
}

Review Article

\author{
V. S. BHUVANESWARI, R. S. CHANDAN* \\ Department of Pharmaceutical Chemistry, JSS College of Pharmacy, JSS Academy of Higher Education and Research, Mysuru \\ Email: rschandan@jssuni.edu.in
}

Received: 22 Jan 2018, Revised and Accepted: 15 Apr 2018

\begin{abstract}
Cosmetics can be defined as, "substances which are intended to be applied to the human body for cleansing, beautifying, promoting attractiveness, or altering the appearance without affecting the body's structure or functions" according to USFDA. This review encompasses a brief description of the process of safety assessment of a cosmetic product. There is no significant systemic absorption of cosmetics by penetration through skin, but some products are intended to apply on the mucous membranes or skin surrounding the mucous membranes which may result in significant systemic absorption on continuous use. Serious health hazards have not been observed mostly, but in some cases, lead to severe reactions like, allergy, irritation, comedogenicity, genotoxicity, photosensitivity etc. At the same time, long terms effects of usage of cosmetics are still unknown as the products are used repeatedly over a long period of time. Hence, safety evaluation of a cosmetic product is the most prominent step before release of the product into the market. There are no specific safety regulations for cosmetics, but the safety of the product is the responsibility of the manufacturer. The article explains about different organizations that are responsible for the safety of the cosmetic products before they are released into the market in different countries and also about the Product Information File (PIF). The safety of a cosmetic product both at industrial level and finished product level are explained. The different tests deployed for the safety assessment of a cosmetic product are also explained.
\end{abstract}

Keywords: Safety of cosmetics, Safety assessment of cosmetic products

(C) 2018 The Authors. Published by Innovare Academic Sciences Pvt Ltd. This is an open access article under the CC BY license (http://creativecommons.org/licenses/by/4.0/) DOI: http://dx.doi.org/10.22159/ijcpr.2018v10i3.27333

\section{INTRODUCTION}

According to USFDA, Cosmetics can be defined as, "substances which are intended to be applied to the human body for cleansing, beautifying, promoting attractiveness, or altering the appearance without affecting the body's structure or functions". So, a cosmetic product can be broadly defined as any material intended for external use as a component of a cosmetic product.

Cosmetic products can be classified based on the area of application on the body i.e., Cosmetics used on

- Face-face powders, compact powders, creams, lotions, sunscreens, rougases, foundation, concealer, lipstick, lip gloss, lip liner, lip plumper etc.

- $\quad$ Eyes-kajal, eye liner, eye lashes, eye shadow, colured contact lenses etc.

- Body-deodorants, skin care lotions, powders, perfumes, bath salts, bath butters etc.

\section{- $\quad$ Baby care products}

- Hands/Nails-nail polish, nailpolish remover, hand sanitizer etc.

- Hair-Hair dyes, hair oils, hair sprays and gels, shampoos, conditioners etc [1].

There is no need for the premarket approval of FDA for the cosmetic products or for the ingredients in United States. FDA only regulates the prevention of usage of the ingredients that are already proved harmful for the human use eg, color additives (manufacturer must use only FDA approved color additives). However, the agency has strictly advised the cosmetic manufacturers to deploy suitable methods for establishing the safety, purity and authenticity of their products and it also states that, the manufacturer is solely responsible for the safety of both the ingredients and the finished cosmetic products prior to marketing [2-3].

There are more stringent regulations in European Union and other agencies around the world. In the EU, manufacturers must be designated as an identified person or a company within the European Union and they have to establish the safety of the product according to the relevant safety requirements under the legislation, then only they can market a cosmetic product [4].

In India, the cosmetic products are regulated by the Central Drug Standard Control Organization (CDSCO) which is overarched by the regulations of Drugs and Cosmetics act, 1940 and 1945. Another agencyin India, named, BIS (Bureau of Indian Standards) issues standards for the ingredients used in the cosmetics. Cosmetic products which are imported from other countries must get registered according Indian legislature, before release into the market. Domestic products doesn't require any registration, but before starting production, the company has to prove that it has suitable facilities, like, factory premises, space, plant and machinery and other requisites for the production and must obtain a license. These are few agencies (but not limited to the above list), which determine the safety, purity, and authenticity of cosmetic products released into the Indian market. Similarly, every agency around the world has its own regulations in establishing the safety of the cosmetic products [5-8].

\section{ICCR and basic steps in safety assessment process}

The International Cooperation on Cosmetics Regulation (ICCR) is an international group of cosmetics regulatory authorities of Brazil, Canada, the European Union, Japan and US. ICCR works voluntarily, and it meets on annual basis to discuss common issues on cosmetic safety and regulation, as well as it enters into a constructive dialogue with relevant cosmetic industry trade associations [9].

ICCR defines safety assessment as: "The safety assessment of cosmetic ingredients is not a standardized procedure, but it should be performed on case-by-case basis using the best science. In particular, checklist approaches and decisions based on hazard alone are considered inappropriate and not adequate to thoroughly assess safety. Safety assessment must involve a systematic stepwise approach that starts from conservative assumptions and include refined approaches, as required and/or appropriate. Safety assessment must utilize the most up-to-date approaches available while taking into account current legal/regulatory requirements."

The basic steps in the safety assessment process are:

- Determination of various ingredients that comprise a product, followed by the ingredient characterization through relevant 
physico-chemical data, purity and profile of impurities and Chemical structure identification

- Based on the literature review or by the previous reports of adverse events, the relevant toxicological information of all the ingredients is assessed. Data on closely related structural analogues and structure-activity modeling data may also be considered for assessment.

- Stability of the ingredients, their microbiological status, and the overall composition of the product is also studied.

- If the formulation consists of an ingredient or particular substance of special concern (i.e., perfumes, UV filters, hair dyes, etc.), it must receive special attention by the safety assessor.

- Profound knowledge on the exposure of the product must be established. i.e., how the product is used, the amount used in one application, frequency of usage, duration of intended use, target users etc.

- Based on exposure data of the product, the 'safety margin' and 'exposure margin' are calculated using the relevant dose.

- After the safety assessment of a product, the conclusions are drawn by the safety assessor, based on which, he may accept, reject, or accept under specific conditions. The recommendations made by the safety assessor, which are relevant for safety-in-use of the product, must be followed up by the responsible person.

- To support the continued safety of the product, post market surveillance is also conducted [10-11].

After completion of the safety assessment, the data is given in the form of, Cosmetic Product Safety Report (CPSR). CPSR is a main part of the Product Information File (PIF) which establishes that that cosmetic product is safe to use.

There are two parts in CPSR, i.e., Part A and Part B

Part A gives all necessary data, which is required to perform product safety assessment. The main sections under Part A are:

1. Quantitative and qualitative composition of the cosmetic product

2. Physical/Chemical characteristics and stability of the cosmetic product

3. Microbiological status of the product

4. Impurities, traces, and information about the packaging materials

5. Normal and reasonably foreseeable use

6. Exposure to the cosmetic product

7. Exposure of the substances

8. Toxicological profile of the substances

9. Undesirable effects and serious undesirable effects

10. Information on the cosmetic product

Part B gives the conclusion of the safety assessment. The main sections under Part B are:

1. Warnings and operating instructions that must be displayed on the label

\section{Scientific reasoning.}

In US and EU, the Product Information File (PIF) is mandatory, in India, there is 'Dossier on cosmetic products', which can be referred as PIF. The concept of PIF is not mandatory in India, but, the manufacturer must be able to prove the safety upon request from authorities.

Cosmetic Product Dossier consists of (but not restricted to)

1. Technical description of the product i.e., the data to be presented on the label and package
2. Ingredient list or the formulation of the product

3. Detailed information on every raw material or ingredients used, including additives and contaminants (if any).

4. Details on compliance with Good Manufacturing Practices (GMP)

5. Product Safety Assessment (PSA)

6. Justification on the established shelf life or PAO (best Period After Opening) and stability of the product

7. Documents pertaining to scientific claims

8. If there are any post marketing data on health related surveillance that shows any undesirable health hazards related to the use of the cosmetic product, then, cosmetovigilance report must also be incorporated

9. Adherence of procedures of preparation and storage of product dossier to the relevant regulatory requirements [11-12].

\section{Safety assessment of a cosmetic product}

Most of the people, especially female's use cosmetic products on a daily basis. Even though, external contact of an ingredient can rarely lead to significant systemic absorption by penetration through the skin, systemic exposure of the cosmetic ingredients can rarely be excluded, because there are some products which are used extensively on oral cavity (face, lips, eyes and mucosa) Eg: kajal, mascara, eye shades, lipsticks, etc. and which may lead to some toxicities like, heavy metal exposure. As there is an extensive and relatively uncontrolled human exposure to cosmetics and their ingredients, these products must be evaluated for their safety, before releasing into market [13].

The safety assessment of a cosmetic product can be conducted at two levels. First level is at the industrial level and the second level is after the production, to make sure that the product produced is safe and ready for release into the market.

\section{Product safety at the Industrial level}

All the products which are manufactured must be fit for the purpose for which it is being manufactured. Cosmetic products, to be fit for their purpose, must be safe and efficient. So, for a product to be fit for the purpose, a good design of the manufacturing procedure is required. This good design will lead to a safe design. Hence a good design must be made and followed through all the stages of design, production, supply and disposal. At the same time, the manufacturing and processing systems must be environmental friendly and must comply with all the technical and legal requirements. If the design is not proper, it may lead to a product of insufficient quality which when used by the consumers, may lead to undesired effects or may not show any effects at all, which in turnmay lead to recall of the product from market. Maintaining the quality, safety and authenticity of the product is the foremost responsibility of the manufacturer.

\section{Specific guidance for cosmetics manufacturers}

1. Documentation: the documentation in the industry should be such that, it must capture every aspect of the manufacturing processes and organizations processes, which prevents errors or interpretation or loss of information. Good documentation allows one to trace the problems which may have occurred during production and to take appropriate corrective actions.

2. Records: Records should be maintained in details for the operations, procedure, deviation from procedures, justifications, instructions, specifications, protocols, reports, methods, precautions, corrections and other measures, and other information which is required by GMP. Records can be very much useful as the proofs of the Quality of the process ex: quality of the raw materials can be verified by raw material records, to evaluate batch production control records to know if the batches are consistent, to evaluate laboratory controls for raw and in-process materials and finished products etc.

3. Buildings and facilities: Buildings and facilities used for manufacturing must be of adequate size, design, and construction 
and must be maintained in a clean and orderly manner. The facilities must provide sufficient space, lighting and ventilation and must be able to prevent cross-contamination, filth and pest control and must ensure the cleanliness of premises and equipment.

4. Equipment: The equipmentand utensils which are used in processing, holding, transferring and packing must have suitable size, design and must be adequate for the intended use. Equipment must be qualified before using and must be calibrated periodically to determine efficiency.

5. Personnel: The person carrying out any operation during manufacturing process must be suitably qualified, trained and experienced to perform their assigned functions.

6. Raw materials: Before using raw materials, it must be determined whether the raw materials are identified, stored, examined, tested, inventoried, handled, and controlled to ensure they conform to appropriate standards and specifications. Some of the raw materials for which special care has to be taken are:

(a) Water: Manufacturer must ensure that, the water used as a cosmetic ingredient

i) is properly treated according to the SOP,

ii) Is of defined quality, or is not affected by materials used in the water treatment equipment

iii) Is being tested regularly to verify that it meets with the specifications of usage.

(b) Color additives: Before using color additives manufacturers must verify if the color additives are certified for use in the desired cosmetic formulation. It should be unlisted in the list of prohibited or restricted cosmetic ingredients and if it's a new color additive manufacturer has to get petition approval from FDA for its use.

7. Production: Manufacturer must ensure that SOPs has been prepared for all the processes i.e., formulation, process instructions, inprocess control methods, finished product analysis methods, packaging and storage instructions etc. and these SOPs are approved for use and followed without any fail by the personnel carrying out the work.

8. Laboratory controls: The laboratory controls include sample collection techniques, specifications, test methods, laboratory equipment and technician qualifications. It must include i) raw material, in-process and finished product examination for quality,

ii) Testing the adequacy of preservation against microbial contamination under reasonable conditions of storage and use of currently finished and retained product samples.

iii) Storage of retained samples under specific conditions to protect its integrity and to retest at periodic intervals to establish its compliance with the laid down specifications

iv) Returned cosmetics are tested for deterioration, contamination and compliance with the specifications

9. Internal audit: internal audits must be conducted regularly or on demand to know about the adherence or compliance of the laid down procedures. They must be conducted by the persons who don't have any direct contact to the data being audited. After the audit, the observations made must be shared with the management and follow-ups must be made to ensure that the observations are followed.

10. Complaints, adverse effects and recalls: The product complaints, consumer adverse event reports and product recall files must be reviewed periodically and required actions must be taken [11-14].

\section{Safety assessment of cosmetic ingredients}

Safety assessment of cosmetic ingredients takes place at the industrial level. For some ingredients safety profile is already established and for the new ones safety profile must be established before using in any formulation.

There is a nonprofit program to assess the safety of ingredients in personal care products, called, Cosmetic Ingredient Review (CIR), which remains the only scientific program in the world committed to systematic, independent review of cosmetic ingredient's safety in a public forum. Its main purpose is to review the ingredients in OverThe-Counter personal care products and it works according to the procedures laid down by USFDA. According to the review of CIR, dated, March-2017, out of 4,740 individual cosmetic ingredients, 4,611 were determined to be safe, 12 determined to be unsafe, and for 117 ingredients, the information available was insufficient to determine its safety. So, before choosing an ingredient, its safety profile and its compatibility with the formulation must be checked [11-15].

Different tests used in the safety assessment of ingredients are:

\section{Physico-chemical characterization:}

- Chemical identity, physical form, molecular weight

- Purity and isomer composition

- Solubility, Partition coefficient

- Characterization of impurities and accompanying contaminants

- Relevant physico-chemical specifications

- Homogeneity and stability

- Functions and uses

2. Toxicological studies:

- Toxicokinetics

- Acute toxicity and

- Local toxicity i.e., Corrosion, irritation and skin sensitization

- Systemic toxicity i.e., dermal absorption, Repeated dose toxicity, Reproductive toxicity, Mutagenicity/genotoxicity, Carcinogenicity

- Photo-induced toxicity [16].

\section{Safety assessment of a finished cosmetic product}

From a recent study, it was found that, an average adult uses nine cosmetic products daily where as a women uses 15 products or more. It was also found that, cosmetics, toiletries, and skin-care products including sunscreens, are the seldom contributors for hospital referrals with allergic contact dermatitis. From this, it is estimated that, $1-3 \%$ of the population are allergic to either cosmetics or their individual ingredients. In the Indian context, incidence of contact allergic dermatitis was observed to be $3.3 \%$ with the use of various cosmetics, and it was observed to be the common adverse effect for cosmetics like, hair dyes, shaving creams and lipsticks in about $59.2 \%$ of population. Photo allergic dermatitis was seen as adverse effect with the use of hair dyes and lipsticks in $35 \%$ of population. Other types of adverse effects can also be observed other than dermatitis with the use of variety of cosmetics. Hence product must be evaluated for safety, to gain the confidence of the consumers.

Safety evaluation of a cosmetic product is performed based on the surface area of the skin exposure of the product per application and number of applications per day and also,type of application. As there is a wide variety of cosmetic products, the exposure scenario can be described as, (but not limited to) e. g:

$>$ Toiletries (Soaps) or skin cleansing agents, which are applied in diluted form where the area of exposure is extensive but the product is rapidly washed off.

$>$ Products which are used on lips and mouth, poses the possibility of ingestion to some extent.

$>$ Products used around eyes and genital regions may come in contact with the conjunctiva or mucosa respectively and may lead to unwanted reactions due to the thin epithelial lining of these areas

$>$ Products like, body lotions or body creams are intended to be used over a large surface of the body leading to the extensive exposure and at the same time prolonged exposure also, as the 
substances of the product, often at appreciable concentrations, may remain in contact with the skin for several hours

> Products like Sunscreens,due to their extensive skin contact and direct exposure to UV radiations for prolonged time, require special and distinct attention during safety evaluation.

Hair dyes also need special attention, because, the substances used in permanent hair dyes undergo oxidation due to hydrogen peroxide present on the hair and the final product of this oxidation, may come in contact with skin, leading to any unwanted reactions.

So, based on the exposure scenario of the product, the amount of substance that may be ingested or inhaled or absorbed through the skin or mucous membrane is calculated and translated into daily amount per Kg body weight, which is considered as, SED (Systemic Exposure Dose) and based on the SED, safety assessment is performed [17].

There are different test procedures deploying, in vivo (both animal and human models) and in vitro models used to determine the safety levels of a cosmetic product. Different tests deployed are (but not restricted to the below list):

1. Patch and photo-patch test: Patch test is conducted to determine the reaction of a particular cosmetic, i.e., if it is an irritant or an allergic. The standard test series can also identify the agents causing allergy which is not identified by blood testing or skin prick testing. In the test, the diluted chemicals are planted on the small area of patient's back and observed for any local allergic reaction. Different types of chemicals include metals (Eg: nickel), rubber, leather, formaldehyde, lanolin etc. For 'leave on' cosmetics Eg: lipsticks, the samples for the test are taken, as is but for 'Wash off' or 'Rinse off cosmetics, eg: shampoos, $10 \%$ solutions are used as samples whereas soaps and detergents, are used in the concentration of $1 \%$. Photo patch test, is conducted to interpret photo dermatitis. Test is considered to be positive if the test site shows dermatitis with the exposure to antigen and sunlight or UV radiations [18].

2. Patch test screening for fragrances/perfumes: The compounds which are marked as recognized markers for fragrance allergy are Balsam of Peru, Cinnamon, fragrance mix and Colophony. Fragrance mix detects about $86 \%$ of positive reactions and it is commercially marketed as, TRUE test kit, (Thin-layer Rapid Use Epicutaneous test) and it contains eugenol, isoeugenol, oak moss absolute, geraniol, amyl cinnamic aldehyde, hydroxyl citronellal cinnamic alcohol, and a-cinnamal. The positive results of fragrance mix can be raised to $96 \%$ with the addition of ylang ylang oil, narcissus oil, sandal wood oil, and balsam of Peru. Allergy to fragrance can also tested by Repeated Open Application Test (ROAT).

3. ROAT (Repeated Open Application Test)/Provocative use test: This test is used to screen for allergy to cosmetics having fragrances and to determine the clinical significance of weak positive patch test reactions. In the test, the suspected chemical substance is applied twice, on a daily basis for up to two weeks on 5 $\mathrm{cm}^{2}$ area of the flexor surface of the forearm. Test is positive, if the rash appears after one week and that particular cosmetic product, is not considered safe where as if rash doesn't appear after one week the test is negative and the product is safe to use for that particular person [19-20].

4. TRUE test (Thin-layer Rapid Use Epicutaneous test): TRUE test is considered as a reliable allergen skin patch test. The test panel contains a negative control, three adhesive panels containing 35 different substances or mixes, all of which are known to cause contact dermatitis. The three adhesive panels are sticked on the upper back of the patient starting from upper left corner. After $48 \mathrm{~h}$ the panels are removed and observed for any allergic reactions. Additional readings at 72 or $96 \mathrm{~h}$ are also taken. Late reactions for the test may also occur [21].

5. Chemical analysis: Sometimes, chemical analysis can also be required to determine the presence of the suspected allergen in the product under test and sometimes, to identify the unknown allergen.
6. Dimethylgloxime test: This is a useful in identifying nickel. This test can be used to identify metallic objects that contain optimum concentration of nickel to provoke an allergic reaction. The test is positive if a red precipitate is obtained, when 1\% Dimethylgloxime and $10 \%$ ammonium hydroxide solution are added to the test solution which indicates that the product is not safe for persons who are hypersensitive to nickel [22].

7. Tests of irritancy and sensitivity: Irritant potential of a chemical is determined or measured using two tests. One is 'Soapchamber test or Use test' and 'Repeat Insult patch test'.

Soap-chamber test is used to determine, if the toiletry/household product is tolerant or irritant. It is also used to compare soaps and detergents which are used on daily basis for relative mild or irritant potentials. Irritation potential can be determined by taking 12-20 individuals and applying the respective patches on the upper back of the individuals and the patches are left undisturbed for $48 \mathrm{~h}$. After $48 \mathrm{~h}$ patches are removed and presence of any reaction is observed. The substance under examination is then reapplied similarly, for further $48 \mathrm{~h}$ in the same area as before. The same procedure is repeated three times a week for about two-three weeks. At the end of two or three weeks patches are removed and the exposed skin is observed for any reactions. This test can be used to determine very low orders of toxicity in a preparation [23].

Human repeat insult patch test (HRIPT) is used to test formulation's irritation and allergy potential. With repeated exposure to a substance, humans can develop sensitization to a substance which on increased exposure may lead to increased allergic responses. So, HRIPT is used to determine sensitization to a particular substance by an individual. For sensitization potential, a larger group of individuals i.e., up to 100 members are taken. Similar procedure as the irritation potential is used, but at the end one more test is added i.e., one week of rest is given for the subjects and the patch of the same substance under study is applied for $48 \mathrm{~h}$ but on different site. After $48 \mathrm{~h}$ if a positive reaction is observed after removing the patch, indicates that the person is sensitized to that particular material [24].

8. Draize eye irritancy test: This test is done to determine the irritancy potential of the products which are applied on the eye or near the eye. In this test, chemical substances (in the concentration of $100 \mathrm{mg} / \mathrm{ml}$ ) are dripped in to the eye of an immobilized, conscious albino rabbit with their eye lids held open with clips. The progressive effects are observed at regular intervals of time up to 72 h. The test may even last up to 7-18 $\mathrm{d}$. The possible reactions by the irritants include swelling of eyelid, inflammation of the iris, ulceration, hemorrhaging, and blindness. But, Draize test is considered crude, imprecise and unreliable because, it doesn't reflect the adequate degree of irritancy in humans [25].

9. Murine Local Lymph Node Assay (LLNA): This test is used to test for substances that possess allergic contact sensitizing potential. The primary purpose of this test is to determine the minimum concentration of chemical substance required to induce allergic stimulation [26].

10. Tests of comedogenicity: USFDA defines a comedogenic ingredient or product as one that is known to clog pores. It clogs pores by increasing the production of keratin in hair follicles leading to clogged follicles which on prolonged exposure leads to comedones. This process is not spontaneous, but may take months to manifest. There is no definite list of comedogenic ingredients, because the comedogenic activity varies with individuals, and the co-ingredients present in a product, i.e., even a non comedogenic product may become comedogenic due to individual's own unique skin. In previous days rabbit's ear test was used to determine the comedogenicity of a product. But, now, this test is conducted on human volunteers. Tests may differ from lab to lab. But, generally 10-30 subjects are taken and respective patches are sticked on their upper back. The results are compared with a positive control and a negative control (untreated sites). At the end of the test period (usually 4-8 w), surface biopsy of the skin is made and observed under magnification. The ingredients are ranked between range 0-5 where 0 is the least or non comedogenic whereas 5 being most 
comedogenic. Ingredients ranked 0-1 showed no significant effect and ingredients ranked, 2-3 resulted in moderate increase in follicular keratosis whereas ingredients ranked 4-5 resulted in an extensive increase in the follicular keratosis. Hence, ingredients that are observed to be comedogenic mustn't be indicated for persons with oily skin or acne prone skin [27].

11. Tests for mutagenicity/genotoxicity: The mutagenic or genotoxic potential of the ingredient is determined by two methods. They are Bacterial reverse mutation test (or in vitro mammalian cell gene mutation test) and in vitro mammalian cell chromosome aberration test.

Bacterial reverse mutation test is used to detect chemicals that cause mutations. If the substance under study is mutagenic, it reverses the mutations already present in the tester strains and restores the capability of the strains to produce an essential amino acid. Salmonella typhimurium and E. coli are suitable for this study. Usually strains which require amino acids for their survival are selected because they help in determining the point mutation. Point mutations may involve substitution, addition, or deletion of one or few DNA base pairs. Test is carried out by dissolving or suspending the test substance in suitable solvent or vehicle and it is diluted suitably before inoculation of bacteria. The results are compared with positive and negative controls. The final data is presented in the form of revertant colonies per plate.

In vitro mammalian cell chromosome aberration test identifies agents which cause structural chromosome aberrations in cultured mammalian cells. Cell cultures are exposed to the test substance with and without metabolic activation. After the exposure, up to suitable period of time, cell cultures are treated with colchicine, harvested (after 3-6 h), strained and metaphase cells are observed microscopically for the presence of chromosome aberrations by using a metaphase-arresting substance such as colcemid. Solid substances under study are dissolved or suspended in suitable solvent or vehicle before starting the test and liquids may be added directly.

12. Tests for photosensitivity: To determine the photosensitive potential of an ingredient or formulation, it must be tested for two types of reactions. i.e.,

a) Phototoxic reactions where the drug or ingredient becomes active on exposure to sunlight (i.e., UV-A exposure and less commonly due to UV-B radiations in sunlight) and causes damage to the skin. The reaction usually clears up once after the drug is discontinued or is cleared from the body. The reaction usually resembles sunburn.

b) Photo allergy reactions, here, exposure of the drug to the UV radiations tends to changes in the drug molecule. This modified drug molecule is recognized as an antigen by the body's immune system leading to an allergic reaction like inflammation and rashes in the exposed area. The reaction usually resembles eczema and it may recur when exposed to sun even after the drug is discontinued or cleared out from the body.

Phototoxicity of all UV absorbing chemicals used in the cosmetics (sun screens etc.) is tested by using in vitro method named, 3T3 Neutral Red Uptake Phototoxicity Test.

\section{In vitro 3T3 neutral red uptake phototoxicity test (3T3 NRU PT)}

Compounds which lead to in vivo photo toxic reactions after application on the skin can be identified by this test. In the test, a permanent mouse fibroblast cell line, Balb/c 3T3, clone 31 is used. Cytotoxicity is measured as a concentration dependent reduction of uptake of the vital dye, Neutral red after 24 hour treatment with the chemical and irradiation to UV radiation. Xenon arc lamp and mercury-metal halide arc lamps are used as a source of UV and visible radiation and to simulate solar light. The test procedure is for three days. On the first day, suitable concentration of cell suspension is prepared and $100 \mu \mathrm{l}$ culture medium is dispensed on to the peripheral walls of a 96-well tissue culture micro titer plate. On the second day cells are incubated with 8 different concentrations of the test chemical in the dark for $60 \mathrm{~min}$ at $7.5 \%$ of carbon dioxide and at $37{ }^{\circ} \mathrm{C}$ temperature. On the third day, the cytotoxic effects of the chemical on the cells are microscopically evaluated by using a phasecontrast microscope and changes in the morphology of the cells are recorded. Results are evaluated by comparing the concentration of the test chemical and inhibition in uptake of $50 \%$ of cellular NRU. This test demonstrated predictive value of $95-100 \%$, hence, is considered a reliable source for the prediction of phototoxicity of an ingredient or a formulation [28-29].

\section{CONCLUSION}

Cosmetics and personal care products have become a necessary tool in beautification and personal health of the human race. There are different types of cosmetics introduced daily into the market for different purposes worldwide. There are no specific safety regulations to establish the safety of a cosmetic product in many countries, and the safety is voluntary and mostly controlled by the manufacturer. At the same time cosmetics are not associated commonly with serious health hazards to humans but their safety must be the principle agenda, because of their regular and prolonged use. Even though they don't pose any serious health effects on regular use, the effect on their prolonged use is still unknown. Cosmetics which incorporates, ingredients whose safety is unclear or ingredients like UV absorbers, dyes, penetration enhancers and ingredients which have limited use, and which tend to pose health risks must be treated with special attention during manufacturing, formulation, and safety assessment to establish the product safety before market release. Even after release of the product into the market, analysis of the products must be conducted periodically to ensure the safety of the product.

\section{ACKNOWLEDGEMENT}

The authors express their gratitude for the Principal, JSS College of Pharmacy, Mysore and JSS Academy of Higher Education and Research, Mysuru for providing spontaneous help and support during the process of writing and publishing the article.

\section{AUTHORS CONTRIBUTIONS}

All the author have contributed equally

\section{CONFLICT OF INTERESTS}

\section{Declared none}

\section{REFERENCES}

1. Cosmetics. Available from: https://en.wikipedia.org/wiki/ Cosmetics. [Last accessed on 10 Dec 2017]

2. FDA regulations on product testing before release into market. Available from: https://www.fda.gov/cosmetics/scienceresearch/producttestin g. [Last accessed on 10 Dec 2017]

3. Regulations on premarket release of cosmetic products in US https://www.fda.gov/Cosmetics/GuidanceRegulation/lawsReg ulations. [Last accessed on 10 Dec 2017]

4. EU Regulations for premarket release of cosmetic products. Available from: http://eur-lex.europa.eu/legal-content. [Last accessed on 10 Dec 2017]

5. Drugs and Cosmetics act; 1940, 1945.

6. Central Drugs Standard Control Organization. Available from: http://www.cdsco.nic.in/forms/Default.aspx. [Last accessed on 10 Dec 2017]

7. Bureau of Indian Standards (BIS). Available from: http://www.bis.gov.in/. [Last accessed on 10 Dec 2017]

8. Indian cosmetic legislation, Chapter 6. Available from: https://cosmetic.chemlinked.com/chempedia/india-cosmeticlegislation. [Last accessed on $10 \mathrm{Dec} 2017$ ]

9. ICCR (International Cooperation on Cosmetic Regulation. Available from: http://www.iccr-cosmetics.org. [Last accessed on 10 Dec 2017]

10. General principles, basic steps in safety assessment of a cosmetic product. Available from: https://www.ecomundo.eu/ en/blog/safety-assessment-cosmetics. [Last accessed on 10 Dec 2017]

11. Guidelines for safety evaluation of finished cosmetic products. Available from: http://ec.europa.eu/health/ scientific_ 
committees/consumer_safety/docs/sccs_o_190.pdf. [Last accessed on 10 Dec 2017]

12. Cosmetics product Dossier. Available from: http:// www.cosmetics-registration-in-india. com/cosmetics-productapplication-dossier-by-regulatory-consultants.html [Last accessed on 10 Dec 2017]

13. Al-Dayel, Hefne J, T Al-Ajyan. Human exposure to heavy metals from cosmetics. Orient J Chem 2011;27:11-101.

14. Guidance for industry. Cosmetic Good Manufacturing Practices; 2013.

15. I J Boyer, W F Bergfeld, B Heldreth, M M Fiume, L I Gill. The cosmetic ingredient review program-expert safety assessments of cosmetic ingredients in an open forum. Int J Toxicol 2017;36(5 Suppl2):13-5.

16. Safety assessment of cosmetics and their ingredients in the EU by Vera Rogiers, Department of toxicology, Vrije Universiteit Brussel, BE; 2017.

17. Nigam PK. Adverse reactions to cosmetics and methods of testing. Indian J Dermatol Venereol Leprol 2009;75:19-10.

18. Patch test, Wikipedia; 2018.

19. (ROAT) Repetitive Open Application Test. Available from: https://www.dermnetnz.org/topics/open-application-test/. [Last accessed on 10 Dec 2017]

20. MHannuksela, H Salo. The repeated open application test (ROAT). Contact Dermatitis 1986;14:221-7.
21. HA Krob, AB Fleischer, RD'agostino, CL Haverstock, S Feldman. Prevalence and relevance of contact dermatitis allergens: a meta-analysis of $15 \mathrm{y}$ of published TRUE test data. J Am Acad Dermatol 2004;51:349-53.

22. RN Shore, S Binnick. Dimethylglyoxime stick test for easier detection of nickel. Arch Dermatol 1977;113:1734.

23. Soap chamber test. Available from: www.albascience.com. [Last accessed on 10 Dec 2017]

24. Human Repeat Insult Patch Test (HRIPT). Available from: www.bioscreen.com. [Last accessed on 10 Dec 2017]

25. JH Draize, G Woodard, HO Calvery. Methods for the study of irritation and toxicity of substances applied topically to the skin and mucous membranes. J Pharmacol Exp Ther 1944;82:377-90.

26. GF Geobrick, CA Ryan, RJ Dearman, I Kimber. Local lymph node assay (LLNA) for detection of sensitization capacity of chemicals. Methods 2007;41:54-60.

27. Comedogenicity-A Complicated Conversation. Available from: www.skininc.com [Last accessed on 10 Dec 2017]

28. Phototoxicity and Photo allergic reactions. www.webmd.com. [Last accessed on 10 Dec 2017]

29. RK Hans, N Agrawal, K Verma, RB Misra, RS Ray, M Farooq. Assessment of the phototoxic potential of cosmetic products. Food Chem Toxicol 2008;46:1653-8. 